\title{
Emergence of HIV drug-resistant mutations in East Indian population after failure of first-line antiretroviral therapy
}

\author{
Nalok Dutta ${ }^{1}$, Srijita Nandi ${ }^{1}$, Subhasish K. Guha ${ }^{2}$, Malay K. Saha ${ }^{1}$ \\ ${ }^{1}$ National Institute of Cholera and Enteric Diseases, Kolkata, West Bengal, India \\ ${ }^{2} \mathrm{~S} c h o o l$ of Tropical Medicine, Kolkata, West Bengal, India
}

\begin{abstract}
Introduction: There are limited data on the failure of first-line antiretroviral therapy (ART) in resourcelimited settings. In particular, there have not been any reports on first-line ART failure among patients in eastern India. We review data on the emergence of human immunodeficiency virus (HIV) drug resistance among individuals with reported first-line ART failure.

Material and methods: Results of genotypic drug resistance testing were obtained from plasma samples of 44 patients who had presented with clinical or immunological failure to treatment after at least six months of ART. Major drug resistance mutations (DRMs) associated with any of the three classes of antiretroviral (ARV) drugs, nucleoside reverse transcriptase inhibitors (NRTI), non-nucleoside reverse transcriptase inhibitors (NNRTI), and protease inhibitors (PI) were seen in all patients (100\% prevalence).

Results: NRTI and NNRTI DRMs were encountered at a frequency of 34 (77.27\%) and 15 (34.09\%) amongst 44 patients, with M184V (34.09\%), T215F (25.0\%), and K219E (20.45\%) being the most frequent among NRTI associated mutations, and Y188L (18.18\%), K103N (6.81\%), and A98G (6.81\%) among NNRTI associated ones. PI DRMs were observed in 5/44 (11.3\%) patients, with V82L, V82S, and I84V being the commonest.

Conclusions: These results present a high prevalence of DRMs among ART patients from eastern India with clinical or immunological failure. It is very important to enhance the access of ARV drugs so that their compliance could be improved and hence development of DRMs be minimised.
\end{abstract}

HIV AIDS Rev 2017; 16, 4: 258-264 DOI: https://doi.org/10.5114/hivar.2017.72027

Key words: antiretroviral therapy, human immunodeficiency syndrome, DRMs, HIV-1, resistance, reverse transcriptase, protease.

\section{Introduction}

India has an estimated population of 2.11 million (1.71-2.64 million) human immunodeficiency virus (HIV)infected individuals with an adult (15-49 years) prevalence of $0.26 \%$ in 2015 [1]. Initiated on April 1, 2004, the program of provision of free antiretroviral therapy (ART) to eligible

Address for correspondence: Dr. Malay K. Saha, National Institute of Cholera and Enteric Diseases P-33, C. I. T. Road,

700010 Kolkata, West Bengal, India, phone: 919433081013 ,

e-mail: sahamk@yahoo.com

individuals has scaled up considerably to register the second largest number of people living with HIV/AIDS (PLHA) across the globe [2]. Compared to 2000 and 2007, the Indian National AIDS Control Program (NACP) has, respectively, achieved $66 \%$ and $32 \%$ decline in new cases of HIV infection in 2015 [1]. With maturing of the program, it is essential to assess the effectiveness of ART by estimating virological

Article history:

Received: 05.10.2017

Received in revised form: 23.11.2017

Accepted: 23.11.2017

Available online: 30.11 .2017
International Journal of HIV-Related Problems

HIV \& AIDS

R e v i e w 
suppression among individuals initiated on a first-line regimen. Concerns over the emergence and spread of HIV drug resistance (HIVDR) also necessitates resistance surveillance studies to monitor treatment outcome [3].

The national program in India implements a public health approach for initiation of first-line ART comprising two nucleoside/nucleotide analogue reverse transcriptase inhibitors (NRTI) and a non-nucleoside reverse transcriptase inhibitor (NNRTI) [4]. The NRTI options available in the program are zidovudine (AZT), abacavir (ABC), lamivudine (3TC), stavudine (d4T), didanosine (DDI), and emtricitabine (FTC), whereas tenofovir (TDF) is the only nucleotide reverse transcriptase inhibitor. The NNRTI options currently available are efavirenz (EFV) and nevirapine (NVP).

In developed nations, viral load (VL) monitoring is a part of the standard treatment protocol, whereas our program still relies on immunological and clinical monitoring, although targeted viral load is offered for confirmation of treatment failure [5]. In the absence of periodic plasma viral load estimation, detection of treatment failure is delayed, which may result in sequential accumulation of resistance mutations, especially thymidine analogue mutations (TAMs) [6]. A large study conducted in Sub-Saharan Africa and Southeast Asia indicated a virological failure (VF) rate of $11.1 \%$ at 12 months of ART [7].

Since the discovery of the virus, the World Health Organization (WHO) and the subsequently created Joint United Nations Programme on HIV/AIDS (UNAIDS) have set goals to control, end, and reduce its spread. The most recent of these goals is 90-90-90: An ambitious treatment target to help end the AIDS epidemic. UNAIDS created the 90-90-90 plan in 2014 as a detailed strategy on how to achieve the Sustainable Development Goals that will eventually end the AIDS epidemic by 2030 (2015).

The management of HIV infection has progressed significantly since the introduction of antiretroviral therapy (cART). Administration of cART durably suppresses HIV replication and is known to reduce both morbidity and mortality associated with HIV infection. A roadblock in the success of cART is the emergence of drug resistance. Transmission of HIV-1 isolates with drug resistance mutations (DRMs) to the next generation of patients can negatively impact prevention programmes and therapeutic strategies. Thus, surveillance of primary DRMs has important public health benefits because the rapid scale-up of ART has been associated with an increase in transmitted DRMs (TDRMs). Since the inception of the National AIDS Control Program in 2004 to provide free cART, India has observed a rapid scale-up of ART access. In resource-rich settings, routine primary drug resistance testing is standard practise for those who are initiating therapy. However, this is not a part of routine standard of care in resource-limited settings like India. Previous studies from India report a variable prevalence of first-line nucleoside reverse transcriptase inhibitors (NRTIs) and non-nucleoside reverse transcriptase inhibitors (NNRTIs) related DRMs in therapy-naive patients ranging between $1.6 \%$ and $38 \%$ [8]. As observed by our group and others, the use of different DRM lists to evaluate the DRM can result in differing prevalence data and can sometimes report falsely elevated prevalence of DRM $[9,10]$. Additionally, other factors that can affect prevalence data include the regional variance of the sampling, subtypes under study and naturally occurring polymorphisms in non-B subtypes. A decade after the ART rollout in India, there is still no archetypal data on primary DRMs in therapy-naive individuals. The last decade has seen a $50 \%$ decline in the number of new HIV infections. While the National AIDS Control Organisation (NACO) estimated that 2.39 million people live with HIV/AIDS in India in 2008-09, a more recent investigation by the Million Death Study Collaborators in the British Medical Journal (2010) estimates the population to be between 1.4-1.6 million people. India has demonstrated a $57 \%$ reduction in estimated annual new HIV infections (among the adult population) from 0.274 million in 2000 to 0.116 million in 2011, and the estimated number of people living with HIV was 2.08 million in 2011. But over the years patients with antiretroviral treatment (ART) failure are increasingly encountered in resource-limited settings [11-13].

Literature studies show $10 \%$ of patients initiated on cART develop some kind of genotypic drug resistance after two years. About 30\% develop viral failure with at least one major resistance mutation within six years [14]. Till date, six years since the commencement of government sponsored ART in India, a significant proportion of the cART-treated $\mathrm{HIV}$-infected Indian population experience ARV failure due to development of HIV DRMs. There have not been any reports on first-line ART failure among patients in West Bengal, eastern India. We review data on the emergence of HIV drug resistance among individuals with reported first-line ART failure. We are the first to analyse the clinical and resistance outcomes of such studies in eastern India $[15,16]$.

Commonly reported HIV DRMs from India are: M184V and L74V followed by the thymidine analogue mutations (TAMs), M41L, K219E, D67N, and T215S for Nucleoside Reverse Transcriptase Inhibitor (NRTI) resistance; Y181C, K103N, V106M, G190A, K101E, and Y188C for Non-Nucleoside Reverse Transcriptase Inhibitor (NNRTI) resistance; and M46I, I47A, V82I, and L90M for Protease Inhibitor (PI) resistance $[17,18]$. This data is a reflection of the HIV populations in the north-eastern part of India. There are few studies that have worked with first-line ART patients in eastern India. Therefore, the aim of the present study is to determine the pattern of HIV DRMs in a large number of cART-treated patients in this region, which could have a bearing on the future planning for ART drug procurement and distribution by the government agencies.

\section{Material and methods}

\section{Patient population}

All HIV-infected patients visiting the Art centre Kolkata were screened for eligibility. They were confirmed for HIV-1 seropositivity by three sets of enzyme-linked 
immunosorbent assay (ELISA) according to NACO guidelines $[19,20]$. Detailed treatment history was taken from all patients. Those who reported no prior exposure to antiretroviral drugs were considered ART naïve. Only adult (> 18 years of age) patients with CD4 cell counts below 200/ $\mu \mathrm{l}$ were considered for entry into the study. The study was conducted following ethical guidelines and written informed consent was taken from all study participants. Out of a starting population of 57 individuals who were considered for entry into the study, 13 individuals did not follow-up during the course of the study and so were discarded. The remaining 44 samples, which were recruited into our study, received first-line ART drug regimen from January 2013 to December 2015. Screening for the enrolled patients were conducted for every six months starting from January 2013 to December 2015. Only patients with HIV viral load > 1000 RNA copies/ml were considered eligible for genotypic drug resistance testing.

\section{Specimens}

Ten millilitres of whole blood sample were taken from each patient. Three millilitres were used for CD4+ T-cell count estimation, and the remaining was centrifuged within six hours of collection at $400 \mathrm{~g}$ for 10 minutes in order to separate plasma. Plasma was distributed into 1-ml aliquots and stored frozen at $-70^{\circ} \mathrm{C}$. Fresh aliquots of plasma were used for HIV-1 RNA quantification and HIV-1 genotyping according to WHO and HIVResNet Laboratory Working Group resistance testing guidelines [21].

\section{HIV-1 genotyping}

HIV-1 genotyping was performed using the Abbott ViroSeq HIV-1 Genotyping Systems (Abbott diagnostics, Wiesbaden, Germany) to sequence the $1.8 \mathrm{~kb}$ protease-RT region of the HIV-1 pol gene as per standard procedure. RNA extraction was performed on $500 \mu \mathrm{l}$ of plasma using the guanidine-thiocyanate extraction method. A reverse transcription polymerase chain reaction (RT-PCR) followed by PCR was carried out to generate an amplicon of $1.3 \mathrm{~kb}$. The amplicons were purified using silica spin columns, and PCR products were run on $1 \%$ agarose gel against two mass ladders allowing for semi-quantitation of DNA. For sequencing, DNA was diluted according the band intensity on agarose gel, and PCR product bands with DNA > 20 nanograms were selected. The latter were added to a 96-well reaction plate containing premixed Big Dye sequencing primers A, B, C, F, G, and H [21]. Sequencing was indigenously carried out in the National Institute of Cholera and Enteric Diseases, Department of Virology on a 16-capillary automated ABI PRISM 3100xl Genetic Analyser (Applied Biosystems, Foster City, CA, USA) using data collection software v3.0 and sequence analysis software v5.3. ViroSeq HIV-1 Genotyping System Software v2.8 was used to assemble the chromatographs from the seven primers into a single project, and to generate a contiguous sequence spanning the entire protease gene and up to codon 335 of the reverse transcriptase (RT) gene. This consensus sequence was compared to a known reference strain, HXB-2, to identify points of variance. The sequences were manually edited and saved in FASTA format, which was submitted to Stanford HIV RT and Protease sequence database [22] to determine the drug-resistance profile and subtype of each sample. DRMs were defined according to WHO Surveillance mutation list 2009 proposed by Bennett et al. [23].

\section{Quality control}

For quality control of HIV-1 genotyping, negative, low-positive, and high-positive control samples were run with every batch. The positive controls ensured the RT-PCR and genotyping success. To ensure good sequence quality, the high-positive control was sequenced before genotyping the HIV-1 clinical samples, precluding editing mistakes.

\section{Clade typing}

HIV-1 subtype was defined using the REGA HIV-1 subtyping tool from the Stanford HIV drug-resistance database (http://hivdb.stanford.edu/). Worldwide subtype references were obtained from the Los Alamos HIV database.

\section{Statistical analysis}

Data was first recorded on a predesigned paper form and subsequently transferred to a Microsoft Excel spreadsheet. All the entries were checked for possible keyboard error(s) at the entry level. The electronic data were exported into the Microsoft Excel spreadsheet, for statistical analysis. Baseline clinical and biological characters of the study subjects were summarised as frequency (\%) for the categorical variables, and mean \pm standard deviation $(\mathrm{SD})$ or median (interquartile range - IQR) for quantitative variables.

\section{Phylogenetic tree}

Clade typing and phylogenetic reconstructions were performed using the REGA sub-typing tool of the HIV drug resistance database, and nucleotide sequences were aligned using the Clustal W multiple sequence alignment program. Phylogenetic analysis was conducted using MEGA v3.0 software. The neighbour-joining method and Kimura parameter model were used for tree construction with reliability estimated from 1000 bootstrap replicates [24, 25].

\section{Results}

Genotyping results were obtained for samples from a total of 44 patients. Their important baseline characteristics are summarised in Table 1 . The mean age ( \pm standard deviation - SD) of participants, of which 30 were males 
Table 1. Baseline characteristics of the study participants

\begin{tabular}{|c|c|}
\hline Parameters & Value $(n=44)$ \\
\hline \multicolumn{2}{|l|}{ Age (years) } \\
\hline Mean \pm SD & $41.4 \pm 8.56$ \\
\hline Range & $27-56$ \\
\hline \multicolumn{2}{|l|}{ Sex } \\
\hline Male/female & $30(68 \%) / 14(31.81 \%)$ \\
\hline $\mathrm{BMI}, \mathrm{kg} / \mathrm{m}^{2}$, mean $\pm \mathrm{SD}$ & $20.5 \pm 7.13$ \\
\hline Range & $14-31$ \\
\hline \multicolumn{2}{|l|}{ Risk factors } \\
\hline Heterosexual & 35 (79.54\%) \\
\hline Blood transfusion & $5(11.36 \%)$ \\
\hline Intravenous drug user & 4 (9.09\%) \\
\hline \multicolumn{2}{|l|}{ Educational status } \\
\hline Literate & $27(61.36 \%)$ \\
\hline Illiterate & 17 (38.64\%) \\
\hline \multicolumn{2}{|l|}{ Locality } \\
\hline Urban & 15 (34.09\%) \\
\hline Rural & 29 (65.91\%) \\
\hline \multicolumn{2}{|l|}{ Marital status } \\
\hline Married & $36(81.81 \%)$ \\
\hline Unmarried & $4(9.09 \%)$ \\
\hline Widow & $3(6.81 \%)$ \\
\hline Widower & $1(2.72 \%)$ \\
\hline $\begin{array}{l}\text { Duration of ART, months, median } \\
\text { (range) }\end{array}$ & $34(11-109)$ \\
\hline \multicolumn{2}{|l|}{ History of change of ART, $n$ (\%) } \\
\hline No change & $32(72.7 \%)$ \\
\hline Once & $8(18.1 \%)$ \\
\hline Twice & $4(9.09 \%)$ \\
\hline Median CD4 cell count, cells/ $\mu \mathrm{l}(\mathrm{IQR})$ & $96(45-184)$ \\
\hline Median viral load, $\log _{10}$ copies/ml (IQR) & $5.10(3.37-7.02)$ \\
\hline \multicolumn{2}{|l|}{ Starting regimen of ART, $n$ (\%) } \\
\hline $\mathrm{D} 4 \mathrm{~T}+3 \mathrm{TC}+\mathrm{NVP}$ & $20(45.45 \%)$ \\
\hline$Z D V+3 T C+N V P$ & $18(40.9 \%)$ \\
\hline $\mathrm{D} 4 \mathrm{~T}+3 \mathrm{TC}+\mathrm{EFV}$ & $4(9.09 \%)$ \\
\hline $\mathrm{ZDV}+3 \mathrm{TC}+\mathrm{EFV}$ & $2(4.54 \%)$ \\
\hline \multicolumn{2}{|l|}{ Opportunistic infections, $n$ (\%) } \\
\hline Cryptosporidiosis & $1(2.275 \%)$ \\
\hline Cytomegalovirus & $3(6.81 \%)$ \\
\hline Pulmonary TB & $14(31.81 \%)$ \\
\hline Extrapulmonary TB & $9(20.45)$ \\
\hline $\begin{array}{l}\text { Pneumocystis jirovecii (carinii) } \\
\text { pneumonia (PCP) }\end{array}$ & $1(2.275 \%)$ \\
\hline Herpes zoster & $3(6.81 \%)$ \\
\hline
\end{tabular}

$B M I$ - body mass index, $A R T$ - antiretroviral treatment, D4T - stavudine, $3 T C$ - lamivudine, NVP - nevirapine, ZDV - zidovudine, EFV - efavirenz, $\mathrm{Pl}$ - protease inhibitors, TB - tuberculosis
Table 2. Frequency of major HIV NRTI drug resistance mutations

\begin{tabular}{|c|c|}
\hline Mutation & No. of patients (\%), $n=44$ \\
\hline NRTI DRMs & 34 (77.27) \\
\hline T69R & $2(4.54)$ \\
\hline K70A & $2(4.54)$ \\
\hline M184V & 15 (34.09) \\
\hline L74V & $1(2.27)$ \\
\hline D67N & $2(4.54)$ \\
\hline M41L & $5(11.36)$ \\
\hline D67S & $5(11.36)$ \\
\hline $\mathrm{T} 69 \mathrm{~N}$ & $5(11.36)$ \\
\hline K70R & $5(11.36)$ \\
\hline $\mathrm{T} 215 \mathrm{~F}$ & $11(25.0)$ \\
\hline K219E & $9(20.45)$ \\
\hline V75L & $1(2.27)$ \\
\hline V75I & $2(4.54)$ \\
\hline T69G & $3(6.81)$ \\
\hline NNRTI DRMs & $15(34.09)$ \\
\hline Y181C & $2(4.54)$ \\
\hline V189D & $1(2.27)$ \\
\hline Y188L & $8(18.18)$ \\
\hline K238T & $1(2.27)$ \\
\hline K101E & $2(4.54)$ \\
\hline K103N & $3(6.81)$ \\
\hline G190A & $2(4.54)$ \\
\hline A98G & $3(6.81)$ \\
\hline Y181G & $1(2.27)$ \\
\hline E138A & $1(2.27)$ \\
\hline PI DRMs & $7(15.90)$ \\
\hline V82S & $1(2.27)$ \\
\hline V82L & $3(6.81)$ \\
\hline $184 \mathrm{~V}$ & $3(6.81)$ \\
\hline
\end{tabular}

HIV - human immunodeficiency virus, DRM - drug resistance mutation, $N R T I$ - nucleoside reverse transcriptase inhibitors, NNRTI - non-nucleoside reverse transcriptase inhibitors, $\mathrm{PI}$ - protease inhibitors

and 14 females, was $41.4( \pm 8.56)$ years. Their median duration of ART use was 34 months (range: 11-109 months). The median CD4 count was 96 (range: $45-184$ ) cells $/ \mathrm{mm}^{3}$, and median $\log _{10}$ viral load/ml was 5.10 (range: $3.37-7.02$ ). ART failure was documented for all individuals by their respective ART clinics. First-line antiretroviral drugs used by the Indian national ART programme are zidovudine (ZDV), lamivudine (3TC), stavudine (D4T), nevirapine (NVP), and efavirenz (EFV). The most common ARV regimen patients were taking at the time of enrolment was $\mathrm{ZDV}+3 \mathrm{TC}+$ NVP (45.45\%), followed by D4T + 3TC + NVP (34.09\%), $\mathrm{D} 4 \mathrm{~T}+3 \mathrm{TC}+\mathrm{EFV}$ (9.09\%), and ZDV + 3TC + EFV (4.54\%), respectively. Pulmonary tuberculosis was observed to be 
Table 3. Frequency of drug resistance mutations by drug class

\begin{tabular}{l|c}
\hline Mutation & No. of Patients (\%), $n=44$ \\
\hline No mutation & $4(9.09)$ \\
\hline One class mutations & $2(4.54)$ \\
\hline Only NRTI & $1(2.27)$ \\
\hline Only NNRTI & $1(2.27)$ \\
\hline Only PI & $37(84.09)$ \\
\hline Two class mutations & $30(68.18)$ \\
\hline NRTI + NNRTI & $4(9.09)$ \\
\hline NRTI + PI & $3(6.81)$ \\
\hline NNRTI + PI & $3(6.81)$ \\
\hline Three class mutations &
\end{tabular}

NRTI - nucleoside reverse transcriptase inhibitors, NNRTI - non-nucleoside reverse transcriptase inhibitors, $\mathrm{Pl}$ - protease inhibitors

the most common opportunistic infection in $14(31.81 \%)$ of the study participants, followed by extrapulmonary TB in nine $(20.45 \%)$, herpes zoster infection and cytomegalovirus was encountered in three (6.81\%), and PCP pneumonia and cryptosporidiosis was found in one $(2.275 \%)$ person. A total of $44(100 \%)$ participants had mutations conferring resistance to at least one ARV drug (Table 2). NRTI mutations were seen in $34(77.27 \%)$ patients, and 15 (34.09\%) had NNRTI mutations. PI mutations were seen in five $(11.3 \%)$ individuals. The most common NRTI mutations observed were M184V (34.09\%), T215F (25.0\%), and K219E (20.45\%) and Y188L (18.18\%), K103N (6.81\%), and A98G (6.81\%) among NNRTIassociated ones. PI DRMs were observed in 7/44 (15.9\%) patients, with V82L, V82S, and I84V being the commonest. Only one class resistance was seen in four (9.09\%) individuals, of whom two $(2.3 \%)$ were with an NRTI and one each had an NNRTI and a PI resistance (2.27\%). Two class resistances were seen in $37(79.7 \%)$ persons with combinations of NRTI + NNRTI, NRTI + PI, and NNRTI + PI. A total of 30 (68.18) patients fell in the category of NRTI + NNRTI combination followed by four (9.09\%) and three $(6.81 \%)$ patients, respectively, for NRTI + PI and NNRTI + PI patients. Three $(6.81 \%)$ patients out of a total of 44 had resistance to all three classes of ARV (Table 3). Subtype analysis of the $44 \mathrm{HIV}-1$ isolates revealed subtype $\mathrm{C}$ as the most predominant type. All HIV-1 isolates clustered with the Indian reference subtype C.

\section{Discussion}

Most of the early studies on HIV drug resistance have focused on HIV-1 subtype B from western countries, and there is a paucity of literature on non-B subtypes [26]. In the present study, all the patients were infected with HIV-1 subtype C. All isolates were clustered around the Indian HIV-1 reference strains JN408366.1, JN408394.1, and JN408393.1 from NARI (National Aids Research Institute),
Pune (Fig. 1). It establishes the predominance of HIV-1 $\mathrm{C}$ among the circulating strains in West Bengal, as seen in other parts of the country [26-28]. Therefore, some of the characteristics of drug resistance mutations identified in this study reflect those seen elsewhere with HIV-1 subtype C. Also, as seen in other studies with HIV-1C subtype, M184V and Y188L emerge as the most prevalent NRTI and NNRTI mutations, respectively. This study is one of the few reports from resource-limited settings describing operational feasibility and programmatic challenges in relation to management of patients living with HIV, who have suspected first-line ART failure. There may be additional factors influencing treatment adherence in such patients; however, it would be difficult to comment on these factors on the basis of routine programme data. Incorporation of combinatorial antiretroviral therapy (cART) into clinical practice has resulted in a $60 \%$ to $80 \%$ decline in rates of acquired immunodeficiency syndrome (AIDS) death and hospitalisation.

In India, previous studies on drug resistance surveillance studies among recently diagnosed individuals from Kakinada, Mumbai, and Northern India showed $<5 \%$ DRM prevalence [29-31]. Earlier studies from different parts of India indicate that the overall prevalence of primary DRMs is moderate (5-15\%) [32-34]. The overall prevalence of RT DRM for Indian strains from treatment-naive patients was determined to be $2.5 \%$, and most of our samples and sequences originated from highly HIV-prevalent states of India. There has been a gradual increase in DRM in therapy-naive patients over the past decade, but the overall prevalence has remained below the WHO threshold level of drug resistance $(<5 \%)$. Our study also showed that there has been a significant rise in NRTI mutations and not in NNRTI mutations.

Owing to improvements in the efficacy and availability of antiretroviral drugs and efforts in HIV/AIDS prevention, recent years have seen a decrease in the incidence of HIV-1 infection. However, an adverse consequence of ART is the emergence and selection of antiretroviral resistant mutant variants, a major cause of ART failure in the treatment of AIDS. These variants have become widespread in drug-treated and untreated individuals infected with HIV, and have compromised the therapeutic options in drugnaive infected persons. Transmission of resistant mutants from drug-experienced patients to newly infected drugnaïve individuals was initially noted in developed countries with good access to antiretroviral drugs. The successful rapid scale-up of access to first-line ART in India warrants an urgent need to establish an on-going system of HIV drug resistance surveillance as per the WHO guidelines for HIV-1 drug resistance 'early warning indicators'. A capacity building programme for regional drug resistance monitoring should be prioritised to implement the drug resistance genotyping in the country. This will not only help us to track primary and transmitted drug resistance at the population level, but will also assist in the optimisation of future therapeutic regimens. 


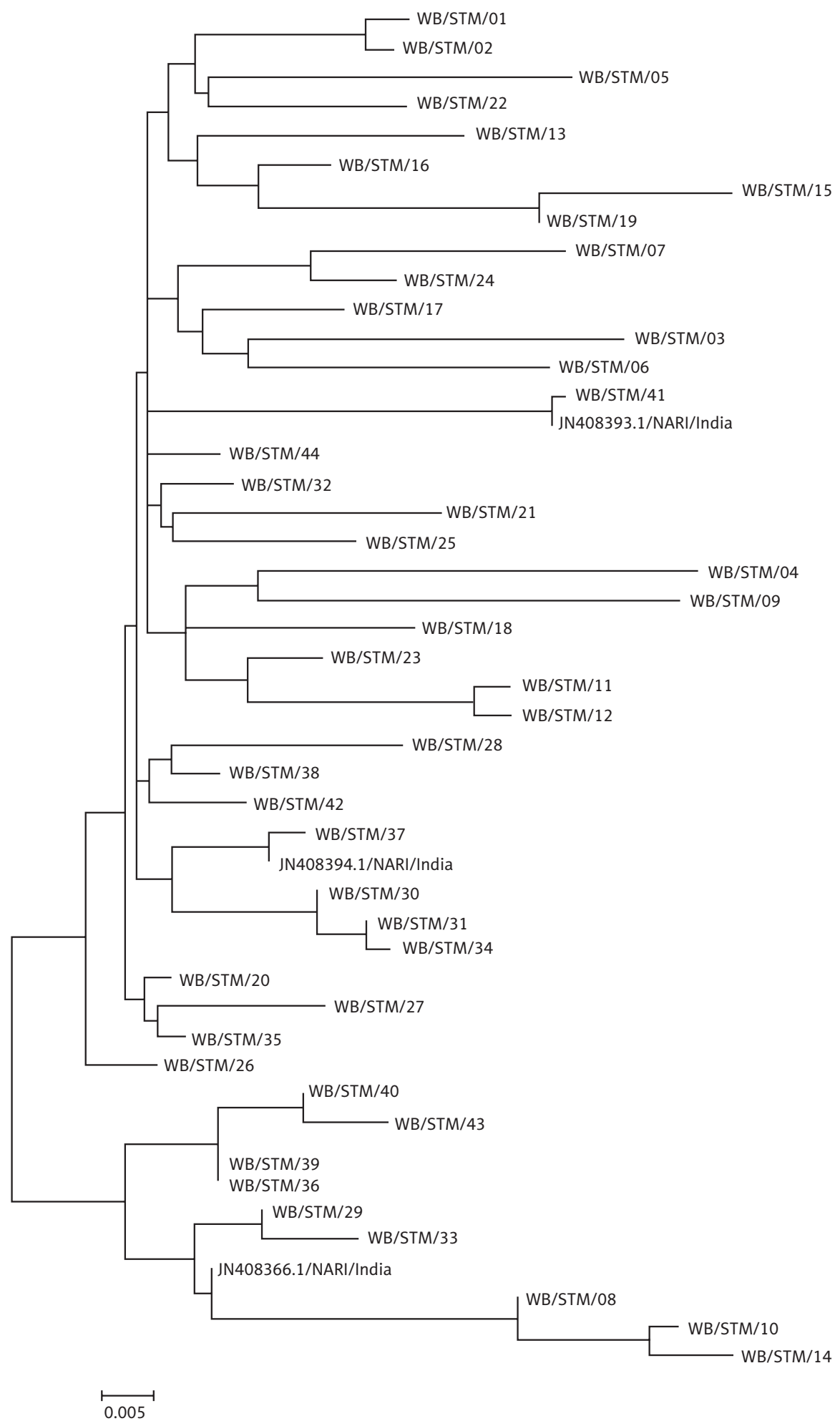

Fig. 1. Molecular phylogenetic analysis by maximum likelihood method. The evolutionary history was inferred by using the Maximum Likelihood method based on the Tamura-Nei model. The tree with the highest log likelihood ( -5348.7146$)$ is shown. Initial tree(s) for the heuristic search were obtained automatically by applying Neighbour-Join and BioNJ algorithms to a matrix of pairwise distances estimated using the Maximum Composite Likelihood ( $\mathrm{MCL}$ ) approach, and then selecting the topology with superior log likelihood value. The tree is drawn to scale, with branch lengths measured in the number of substitutions per site. The analysis involved 47 nucleotide sequences. Codon positions included were $1^{\text {st }}+2^{\text {nd }}+3^{\text {rd }}+$ Noncoding. All positions containing gaps and missing data were eliminated. There were a total of 887 positions in the final dataset. Evolutionary analyses were conducted in MEGA6 


\section{Conflict of interest}

The authors declare no potential conflicts of interest with respect to the research, authorship, and/or publication of this article.

\section{References}

1. National AIDS Control Organization and National Institute of Medical Statistics (ICMR). India HIV Estimations 2015. Technical Report. Available at: http://www.naco.gov.in/upload/ 2015\%20MSLNS/HSS/India\%20HIV\%20Estimations\%202015.pdf (Accessed: 1.12.2015).

2. Global Report. UNAIDS report on the global AIDS epidemic 2013. Joint United Nations Programme on HIV/AIDS (UNAIDS), 2013. Available at: http://www.unaids.org/sites/default/files/media_asset/ UNAIDS_Global_Report_2013_en_1.pdf (Accessed: 1.01.2015).

3. Jordan MR, Bennett DE, Wainberg MA, et al. Update on World Health Organization HIV Drug Resistance Prevention and Assessment Strategy: 2004-2011. Clin Infect Dis 2012; 54 Suppl 4: S245-S249.

4. National AIDS Control Organization, Department of AIDS Control. Anti Retroviral Therapy Guidelines for HIV-Infected Adults and Adolescents, May 2013. Available at: http://www.naco.gov.in/ NACO/About_NACO/Policy_Guidelines/Policies_Guidelines1/ (Accessed: 1.01.2015)

5. National AIDS Control Organization, Department of AIDS Control, Ministry of Health and Family Welfare. Government of India. Annual report 2014-15. Available at: http://www.naco.gov.in/ upload/2015\%20MSLNS/Annual\%20report\%20_NACO_2014-15. pdf (Accessed: 1.12.2015).

6. Barth RE, Aitken SC, Tempelman H, et al. Accumulation of drug resistance and loss of therapeutic options precede commonly used criteria for treatment failure in HIV-1 subtype-C-infected patients. Antivir Ther 2012; 17: 377-386.

7. Aghokeng AF, Monleau M, Eymard-Duvernay S, et al. Extraordinary heterogeneity of virological outcomes in patients receiving highly antiretroviral therapy and monitored with the World Health Organization public health approach in sub-Saharan Africa and Southeast Asia. Clin Infect Dis 2014; 58: 99-109.

8. Deshpande A, Karki S, Recordon-Pinson P, et al. Drug resistance mutations in HIV type 1 isolates from naive patients eligible for first line antiretroviral therapy in JJ Hospital, Mumbai, India. AIDS Res Hum Retroviruses 2011; 27: 1345-1347.

9. Neogi U, Sahoo PN, De Costa A, et al. High viremia and low level of transmitted drug resistance in antiretroviral therapy-naive perinatally-infected children and adolescents with HIV-1 subtype C infection. BMC Infect Dis 2012; 12: 317.

10. Sungkanuparph S, Kiertiburanakul S, Sukasem C, et al. Discrepancies between WHO 2009 and IAS-USA 2009 lists for determining the rate of transmitted HIV-1 drug resistance: a prospective study. J Acquir Immune Defic Syndr 2012; 59: e3-e5.

11. Hosseinipour MC, van Oosterhout JJ, Weigel R, et al. The public health approach to identify antiretroviral therapy failure: high-level nucleoside reverse transcriptase inhibitor resistance among Malawians failing first-line antiretroviral therapy. AIDS 2009; 23: 1127-1134.

12. Leger P, Charles M, Severe P, et al. 5-year survival of patients with AIDS receiving antiretroviral therapy in Haiti. N Engl J Med 2009; 361: 828-829.

13. Hamers R, Wallis CL, Kityo C, et al. HIV-1 drug resistance in antiretroviral-naive individuals in sub-Saharan Africa after rollout of antiretroviral therapy: a multicentre observational study. Lancet Infect Dis 2011; 11: 750-759.

14. Gupta R, Hill A, Sawyer AW, et al. Emergence of drug resistance in HIV type 1-infected patients after receipt of first-line highly active antiretroviral therapy: a systemic review of clinical trials. Clin Infect Dis 2008 ; $47: 712-722$.
15. Deshpande A, Recordon-Pinson P, Deshmukh R. Molecular characterization of HIV type 1 isolates from untreated patients of Mumbai (Bombay), India and detection of rare resistance mutations. AIDS Res Hum Retroviruses 2004; 20: 1032-1035.

16. Hira SK, Panchal K, Parmar PA, et al. High resistance to antiretroviral drugs: the Indian experience. Int J STD AIDS 2004; 15: 173-177.

17. Lakshmi R, Ramesh K, Narayanan PR, et al. Antiretroviral drugresistant mutations at baseline and at time of failure of antiretroviral therapy in HIV type 1-coinfected TB patients. AIDS Res Hum Retroviruses 2009; 25: 1179-1185.

18. Lakshmi R, Ramesh K, Hanna LE, et al. Emergence of drug resistant mutations after single dose nevirapine exposure in HIV-1 infected pregnant women in south India. Indian J Med Res 2010; 132: 509-512.

19. NACO, HIV Testing policy and functioning of VCTC. Available at: www.nacoonline.org.

20. NACO, HIV Testing manual: Laboratory Diagnosis, Biosafety and Quality Control. Available at: www.nacoonline.org.

21. Eshleman H, Hackett J, Swanson P, et al. Performance of the Celera Diagnostics ViroSeq HIV-1 genotyping system for sequence-based analysis of diverse human immunodeficiency virus type 1 strains. J Clin Microbiol 2004; 42: 2711-2717.

22. Stanford University HIV Drug Resistance Database IVDR). Available at: http://hivdb.stanford.edu.

23. Bennett DE, Camacho RJ, Otelea D, et al. Drug resistance mutations for surveillance of transmitted HIV-1 drug-resistance: 2009 update. PLoS One 2009; 4: e4724.

24. Thompson JD, Higgins DG, Gibson TJ. CLUSTAL W: improving the sensitivity of progressive multiple sequence weighting, position specific alignment through sequence weight matrix choice. Nucleic Acids Res 1994; 22: 4673-4680.

25. Kumar S, Tamura K, Nei M. MEGA3: Integrated software for molecular evolutionary genetic analysis and sequence alignment. Brief Bioinform 2004; 5: 150-163.

26. Kantor R, Katzenstein D. Drug resistance in non-subtype B HIV-1. J Clin Virol 2004; 29: 152-159.

27. Khan IF, Vajpayee M, Prasad VS, et al. Genetic diversity of HIV type 1 subtype $\mathrm{C}$ env gene from India. AIDS Res Hum Retroviruses 2007; 23: 934-940.

28. Gadkari DA, Moore D, Sheppard HW, et al. Transmission of genetically diverse strains of HIV-1 in Pune, India. Indian J Med Res 1998; 107: 1-9.

29. Thorat SR, Chaturbhuj DN, Hingankar NK, et al. Surveillance of transmitted HIV type 1 drug resistance among HIV type 1-positive women attending an antenatal clinic in Kakinada, India. AIDS Res Hum Retroviruses 2011; 27: 1291-1297.

30. Sinha S, Ahmad H, Shekhar RC, et al. Prevalence of HIV drug resistance mutations in HIV type 1 isolates in antiretroviral therapy naive population from northern India. Aids Res Treat 2012; 2012: 905823.

31. Chaturbhuj DN, Hingankar NK, Srikantiah P, et al. Transmitted HIV drug resistance among HIV-infected voluntary counseling and testing centers (VCTC) clients in Mumbai, India. AIDS Res Hum Retroviruses 2010; 26: 927-932.

32. Arora SK, Gupta S, Toor JS, et al. Drug resistance associated genotypic alterations in the pol gene of HIV type 1 isolates in ART-naive individuals in North India. AIDS Res Hum Retroviruses 2008; 24 : 125-130.

33. Lall M, Gupta RM, Sen S, et al. Profile of primary resistance in HIV-1infected treatment-naive individuals from Western India. AIDS Res Hum Retroviruses 2008; 24: 987-990.

34. Iqbal HS, Solomon SS, Madhavan V, et al. Primary HIV-1 drug resistance and polymorphic patterns among injecting drug users (IDUs) in Chennai, Southern India. J Int Assoc Physicians AIDS Care (Chic) 2009; 8: 323-327. 